\title{
Anomalous Left Main Coronary Artery Originating from the Right Sinus of Valsalva
}

\author{
Silpa Yalamanchili, MS IV. Kevin M Curl, MD
}

\section{INTRODUCTION}

An anomalous left main coronary artery is a rarely seen clinical entity, particularly when it arises from the right sinus of Valsalva. This case report highlights this uncommon finding and how it affects the care of a patient with significant coronary artery disease.

\section{CASE PRESENTATION}

A 66 year-old male with a history of hypertension, hyperlipidemia, and type II diabetes presented with progressive exertional mid-epigastric and mid-chest discomfort. The patient stated that he had been feeling this "heaviness" with various activities and occasionally at rest for the past nine months. When it occurred with activity, the pain was generally relieved by rest within several minutes. His medications on presentation included insulin glargine, glyburide, metformin, simvastatin, pioglitazone, and lisinopril. Vital signs at the time of presentation included a temperature of $98.2^{\circ} \mathrm{F}$, heart rate of 82 beats per minute, and blood pressure of $130 / 70 \mathrm{mmHg}$ in both arms.

Laboratory investigation showed a total cholesterol of $178 \mathrm{mg} / \mathrm{dL}$ (normal range = 150-250), high-density lipoprotein (HDL) cholesterol of $42 \mathrm{mg} / \mathrm{dL}$, low-density lipoprotein (LDL) cholesterol of $111 \mathrm{mg} / \mathrm{dL}$, triglycerides of $123 \mathrm{mg} / \mathrm{dL}$, and hemoglobin A1C of $7.9 \%$ (normal range $=<5.7 \%$ ). Pharmacologic nuclear stress testing revealed a severe, medium sized defect in the inferolateral wall that was predominately reversible. Thus, the patient underwent a cardiac catheterization which revealed an anomalous left main (LM) coronary artery arising from the right sinus of Valsalva separately from the origin of the right coronary artery (RCA). The distal left anterior descending artery (LAD) had a total occlusion, while the RCA had several areas of $70 \%$ stenosis. A subsequent coronary CT scan displayed an anomalous left coronary artery coursing anterior to the pulmonary artery (Figures 1, 2). The CT scan also showed moderate to high grade RCA stenosis in the mid to distal area of the vessel, as well as high-grade stenoses in the small-sized LAD and left circumflex arteries.

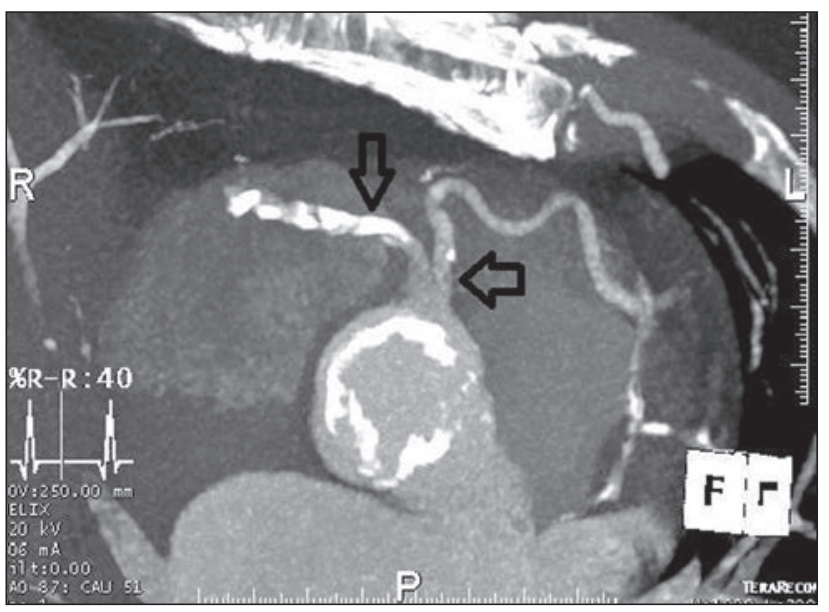

Figure 1. Coronary CT scan showing the separate origins of both the RCA (vertical arrow) and LM (horizontal arrow) from the right sinus of Valsalva.

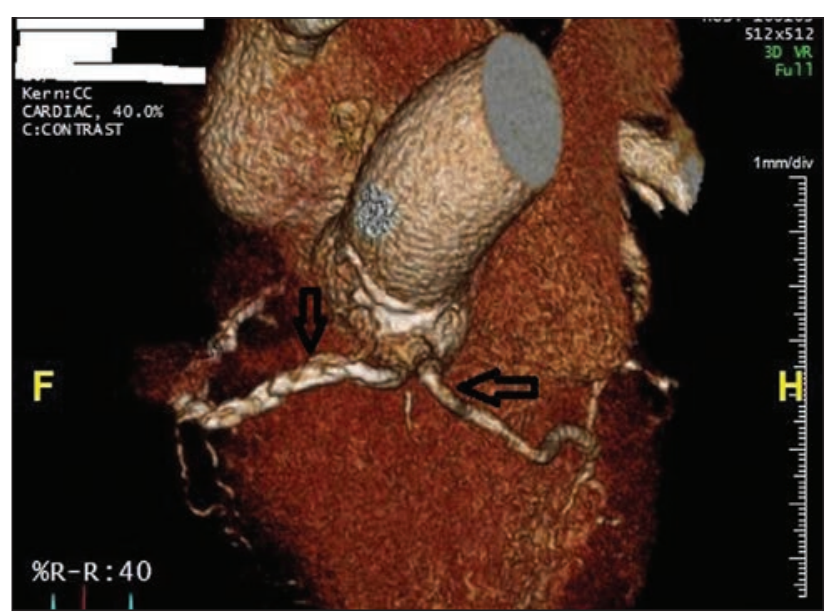

Figure 2. 3D coronary $C T$ scan reconstruction again demonstrating dual origins of the RCA (vertical arrow) and LM (horizontal arrow) from the right sinus of Valsalva. 


\section{OUTCOME AND FOLLOW-UP}

This management of this patient is currently being discussed by a multidisciplinary team including his primary care provider, clinical cardiologist, interventional cardiologist and a cardiothoracic surgeon. It is felt that his anomalous coronary artery was an incidental finding and his uncontrolled medical co-morbidities have led to significant obstructive coronary disease causing his symptoms. Thus, aggressive medical management has been implemented while the patient's long term options are being reviewed, with a significant focus on tighter diabetic and lipid control.

\section{DISCUSSION}

An aberrant left main coronary artery originating from the right sinus of Valsalva is an extremely rare occurrence, with an incidence of $0.06 \%$ to $0.19 \%$ by angiographic study. ${ }^{1}$ In such cases, the anomalous LM is classified into one of four categories based on its course in relation to the aorta and pulmonary trunk: (1) posterior or retroaortic; passing behind the aortic root, (2) interarterial or preaortic; passing between the aorta and pulmonary trunk, (3) anterior or prepulmonic; passing in front of the pulmonary trunk, or (4) septal or subpulmonic; passing within the interventricular septum, beneath the pulmonary trunk. ${ }^{2}$ Of the four courses, an anomalous LM with septal course is the most common, while an anterior LM course is the least common.

While an anomalous LM with interarterial course is associated with myocardial ischemia or sudden cardiac death in young individuals, ${ }^{1}$ an aberrant LM with an anterior, posterior, or septal course is generally considered benign. ${ }^{3}$ Clinically significant symptoms associated with an anterior course are uncommon, but have been reported in two cases. ${ }^{4}$ The most common symptoms of this variant include angina pectoris and myocardial ischemia in the absence of coronary artery disease.
Diagnostic evaluation of asymptomatic patients in which an anomalous LM is suspected is currently indicated only for those at highest risk of sudden cardiac death: young athletes and military personnel. In patients who present symptomatically, CT angiography is able to detect coronary anomalies nearly as effectively as coronary angiography. ${ }^{5}$

Symptomatic patients or young athletes with anomalous LM typically warrant surgical intervention, either by local repair of the anomalous segment or by coronary artery bypass and graft (CABG). ${ }^{5}$ Other treatment options in these patients include medical management with beta blockers or coronary angioplasty with stent deployment.

\section{KEY POINTS}

Anomalous left main coronary arteries, although rare, can complicate the care of patients with clinically significant obstructive coronary artery disease.

\section{REFERENCES}

1. Angelini, P. Congenital heart disease for the adult cardiologist. Circulation, 2007; 115: 1296-1305

2. Ropers, D. Anomalous course of the left main or left anterior descending coronary artery originating from the right sinus of Valsalva. Circulation. 2002; 105: e42-e43.

3. Okuyan, E. Left main coronary artery arising from right sinus of Valsalva: a rare congenital anomaly associated with distal vasospasm. Kardiol Pol. 2011; 69, 5: 505-506.

4. Chaitman, BR. Clinical, angiographic, and hemodynamic findings in patients with anomalous origin of the coronary arteries. Circulation. 1976; 53: 122-131.

5. Angelini, P. Symptomatic anomalous origination of the left coronary artery from the opposite sinus of Valsalva. Texas Heart Institute Journal. 2006; 33(2): 171-179. 